\title{
Sharp generalized Papenfuss-Bach-type inequality
}

\section{Ling Zhu}

Department of Mathematics, Zhejiang Gongshang University, Hangzhou, China.

Communicated by M. Bohner

\begin{abstract}
In this paper, we prove and develop a conjecture on the generalized double Papenfuss-Bach inequality proposed by Sun and Zhu [Z. Sun, L. Zhu, J. Appl. Math., 2011 (2011), 9 pages]. In the last section we pose a conjecture on a general form of Papenfuss-Bach-type inequality.
\end{abstract}

Keywords: Circular approximation, Bernoulli numbers, Papenfuss-Bach inequality.

2010 MSC: 33B10, 26 D05.

\section{Introduction}

Papenfuss [18] proposed an open problem described as follows.

Problem 1.1. Let $0 \leqslant x<\pi / 2$. Then

$$
x \sec ^{2} x-\tan x \leqslant \frac{8 \pi^{2} x^{3}}{\left(\pi^{2}-4 x^{2}\right)^{2}} .
$$

Bach [2] confirmed Problem 1.1 and obtained a further result.

Theorem 1.2. Let $0 \leqslant x<\pi / 2$. Then

$$
x \sec ^{2} x-\tan x \leqslant \frac{2 \pi^{2}}{3} \frac{x^{3}}{\left(\pi^{2}-4 x^{2}\right)^{2}} .
$$

Ge [5] obtained a lower bound of the above inequality as follows.

Theorem 1.3. Let $0<x<\pi / 2$. Then

$$
\frac{64 x^{3}}{\left(\pi^{2}-4 x^{2}\right)^{2}}<x \sec ^{2} x-\tan x<\frac{2 \pi^{4}}{3} \frac{x^{3}}{\left(\pi^{2}-4 x^{2}\right)^{2}}
$$

Email address: zhuling05710163.com (Ling Zhu)

doi: $10.22436 /$ jnsa.011.06.04

Received: 2017-11-08 Revised: 2018-03-16 Accepted: 2018-03-22 
Sun and Zhu [17] gave the better bounds for Papenfuss-Bach inequality above as in the following statement.

Theorem 1.4. Let $0<x<\pi / 2$. Then

$$
\frac{\frac{2 \pi^{4}}{3} \chi^{3}+\left(\frac{8 \pi^{4}}{15}-\frac{16 \pi^{2}}{3}\right) x^{5}}{\left(\pi^{2}-4 \chi^{2}\right)^{2}}<x \sec ^{2} x-\tan x<\frac{\frac{2 \pi^{4}}{3} \chi^{3}+\left(\frac{256}{\pi^{2}} \cdot \frac{513}{511}-\frac{8 \pi^{2}}{3}\right) \chi^{5}}{\left(\pi^{2}-4 \chi^{2}\right)^{2}} .
$$

In the last section in [17], Sun and Zhu posed the following problem.

Problem 1.5. Let $0<x<\pi / 2$. Then the double inequality

$$
\frac{\frac{2 \pi^{4}}{3} \chi^{3}+\left(\frac{8 \pi^{4}}{15}-\frac{16 \pi^{2}}{3}\right) \chi^{5}}{\left(\pi^{2}-4 \chi^{2}\right)^{2}}<x \sec ^{2} \chi-\tan x<\frac{\frac{2 \pi^{4}}{3} \chi^{3}+\left(\frac{256}{\pi^{2}}-\frac{8 \pi^{2}}{3}\right) \chi^{5}}{\left(\pi^{2}-4 \chi^{2}\right)^{2}}
$$

holds, where $\left(8 \pi^{4} / 15-16 \pi^{2} / 3\right)$ and $\left(256 / \pi^{2}-8 \pi^{2} / 3\right)$ are the best constants in (1.1).

By using an automated proof of mixed circular inequalities, Malesevic and Markagic [12] proved Problem 1.5 while Chen and Paris [4] also affirmed Problem 1.5 using a series representation of the remainder in a different expansion for the function $\left(x \sec ^{2} x-\tan x\right)$.

The first purpose of this paper is to show a simple proof of the double inequality (1.1), the second objective is to extend the above conclusions and give further results as follows.

Theorem 1.6. The double inequality

$$
\frac{34 \pi^{4}-448 \pi^{2}+1120}{105}<\frac{\left(\pi^{2}-4 x^{2}\right)^{2}\left(x \sec ^{2} x-\tan x\right)-\left(\frac{2 \pi^{4}}{3} x^{3}+\left(\frac{8 \pi^{4}}{15}-\frac{16 \pi^{2}}{3}\right) x^{5}\right)}{x^{7}}<\frac{160 \pi^{4}-32 \pi^{6}+15360}{15 \pi^{4}}
$$

holds for all $x \in(0, \pi / 2)$, where the lower and upper bounds are sharp.

This paper utilizes the series expansions of certain functions, the properties of Bernoulli numbers, and the relationship between Bernoulli numbers, to achieve the above described. In the last section we pose a conjecture on a general form of Papenfuss-Bach-type inequality.

\section{Lemmas}

Lemma 2.1 ([3]). Let $a_{n}$ and $b_{n}(n=0,1,2, \cdots)$ be real numbers, and let the power series $A(x)=\sum_{n=0}^{\infty} a_{n} x^{n}$ and $\mathrm{B}(\mathrm{x})=\sum_{n=0}^{\infty} \mathrm{b}_{\mathrm{n}} \mathrm{x}^{\mathrm{n}}$ be convergent for $|x|<\mathrm{R}(\mathrm{R} \leqslant+\infty)$. If $\mathrm{b}_{\mathrm{n}}>0$ for $\mathrm{n}=0,1,2, \cdots$, and if $\varepsilon_{\mathrm{n}}=\mathrm{a}_{\mathrm{n}} / \mathrm{b}_{\mathrm{n}}$ is strictly increasing (or decreasing) for $\mathrm{n}=0,1,2, \cdots$, then the function $\mathrm{A}(\mathrm{x}) / \mathrm{B}(\mathrm{x})$ is strictly increasing (or decreasing $)$ on $(0, R)(R \leqslant+\infty)$.

Lemma 2.2 ([1]). Let $\mathrm{B}_{2 \mathrm{n}}$ be the even-indexed Bernoulli numbers. Then

$$
\frac{2(2 n) !}{(2 \pi)^{2 n}}<\left|B_{2 n}\right|<\frac{2(2 n) !}{(2 \pi)^{2 n}}\left(\frac{1}{1-2^{1-2 n}}\right)
$$

Lemma 2.3 ([16]). For $\mathrm{n} \in \mathbb{N}$, Bernoulli numbers $\mathrm{B}_{2 \mathrm{n}}$ satisfy

$$
\frac{2^{2 n-1}-1}{2^{2 n+1}-1} \frac{(2 n+1)(2 n+2)}{\pi^{2}}<\left|\frac{B_{2 n+2}}{B_{2 n}}\right|<\frac{\left(2^{2 n}-1\right)}{\left(2^{2 n+2}-1\right)} \frac{(2 n+1)(2 n+2)}{\pi^{2}}
$$


Lemma 2.4. Let $\mathrm{B}_{2 \mathrm{n}}$ be the even-indexed Bernoulli numbers, we have the following power series expansions

$$
\begin{aligned}
\tan x & =\sum_{n=1}^{\infty} \frac{2^{2 n}-1}{(2 n) !} 2^{2 n}\left|B_{2 n}\right| x^{2 n-1}, \\
\sec ^{2} x & =\sum_{n=1}^{\infty} \frac{2^{2 n}\left(2^{2 n}-1\right)(2 n-1)}{(2 n) !}\left|B_{2 n}\right| x^{2 n-2}
\end{aligned}
$$

hold for all $x \in(-\pi / 2, \pi / 2)$.

Proof. The power series expansions (2.2) can be found in $[6,1.3 .1 .4(3)]$. By (2.2), we have

$$
\sec ^{2} x=(\tan x)^{\prime}=\sum_{n=1}^{\infty} \frac{2^{2 n}\left(2^{2 n}-1\right)(2 n-1)}{(2 n) !}\left|B_{2 n}\right| x^{2 n-2}
$$

\section{Solving Problem 1.5 in a simple way}

Let

$$
h(x)=\frac{\frac{2}{3} \pi^{4} x^{3}-\left(\pi^{2}-4 x^{2}\right)^{2}\left(x \sec ^{2} x-\tan x\right)}{x^{5}} .
$$

Then we can write $h(x)$ as

$$
h(x)=\frac{\frac{\frac{2}{3} \pi^{4} x^{3}}{\left(\pi^{2}-4 x^{2}\right)^{2}}-\left(x \sec ^{2} x-\tan x\right)}{\frac{x^{5}}{\left(\pi^{2}-4 x^{2}\right)^{2}}}:=\frac{h_{1}(x)}{h_{2}(x)} .
$$

Expanding $h_{i}(x)(i=1,2)$ in power series yields

$$
\begin{aligned}
h_{1}(x) & =\frac{\frac{2}{3} \pi^{4} x^{3}}{\left(\pi^{2}-4 x^{2}\right)^{2}}-\left(x \sec ^{2} x-\tan x\right) \\
& =\frac{2}{3} \pi^{4} x^{3} \sum_{n=1}^{\infty} \frac{4^{n-1} n}{\pi^{2 n+2}} x^{2 n-2}-x^{3} \sum_{n=2}^{\infty} \frac{(n-1)\left(2^{2 n}-1\right) 2^{2 n+1}}{(2 n) !}\left|B_{2 n}\right| x^{2 n-4} \\
& =x^{5} \sum_{n=3}^{\infty}\left(\frac{2}{3} \frac{4^{n-2}(n-1)}{\pi^{2 n-4}}-\frac{(n-1)\left(2^{2 n}-1\right) 2^{2 n+1}}{(2 n) !}\left|B_{2 n}\right|\right) x^{2 n-6} \\
& :=x^{5} \sum_{n=3}^{\infty} u_{n} x^{2 n-6}, \\
h_{2}(x) & =\frac{x^{5}}{\left(\pi^{2}-4 x^{2}\right)^{2}}=x^{5} \sum_{n=3}^{\infty}(n-2) \frac{4^{n-3}}{\pi^{2 n-2}} x^{2 n-6}:=x^{5} \sum_{n=3}^{\infty} v_{n} x^{2 n-6},
\end{aligned}
$$

where

$$
\begin{aligned}
& u_{n}=(n-1)\left(\frac{2}{3} \frac{4^{n-2}}{\pi^{2 n-4}}-\frac{\left(2^{2 n}-1\right) 2^{2 n+1}}{(2 n) !}\left|B_{2 n}\right|\right) \\
& v_{n}=(n-2) \frac{4^{n-3}}{\pi^{2 n-2}}>0 .
\end{aligned}
$$


By Lemma 2.1, to prove $h(x)$ is decreasing on $(0, \pi / 2)$, it suffices to prove $\left\{u_{n} / v_{n}\right\}_{n} \geqslant 3$ is decreasing, which is equivalent to

$$
\begin{aligned}
w_{n}= & u_{n+1}-\frac{v_{n+1}}{v_{n}} u_{n}=n\left(\frac{2}{3} \frac{4^{n-1}}{\pi^{2 n-2}}-\frac{\left(2^{2 n+2}-1\right) 2^{2 n+3}}{(2 n+2) !}\left|B_{2 n+2}\right|\right) \\
& -4 \frac{(n-1)^{2}}{\pi^{2}(n-2)}\left(\frac{2}{3} \frac{4^{n-2}}{\pi^{2 n-4}}-\frac{\left(2^{2 n}-1\right) 2^{2 n+1}}{(2 n) !}\left|B_{2 n}\right|\right) \\
& <0
\end{aligned}
$$

for $n \geqslant 3$. To this end, we write $w_{n}$ as

$$
w_{n}=2^{2 n+3}\left(\frac{(n-1)^{2}\left(2^{2 n}-1\right)}{\pi^{2}(n-2)(2 n) !}-\frac{\left(2^{2 n+2}-1\right) n}{(2 n+2) !} \frac{\left|B_{2 n+2}\right|}{\left|B_{2 n}\right|}\right)\left|B_{2 n}\right|-\frac{2^{2 n}}{6(n-2) \pi^{2 n-2}} .
$$

Using the left-hand side inequality in Lemma 2.3 and the right-hand side one in Lemma 2.2 we give

$$
\begin{aligned}
w_{n} & <2^{2 n+3}\left(\frac{(n-1)^{2}\left(2^{2 n}-1\right)}{\pi^{2}(n-2)(2 n) !}-\frac{n\left(2^{2 n+2}-1\right)\left(2^{2 n-1}-1\right)}{(2 n) !\left(2^{2 n+1}-1\right) \pi^{2}}\right)\left|B_{2 n}\right|-\frac{2^{2 n}}{6(n-2) \pi^{2 n-2}} \\
& =\frac{2^{4 n+2}+3\left(n^{2}-2 n-2\right) 2^{2 n}+2}{(n-2)\left(2^{2 n+1}-1\right)} \frac{2^{2 n+2}}{(2 n) ! \pi^{2}}\left|B_{2 n}\right|-\frac{2^{2 n}}{6(n-2) \pi^{2 n-2}} \\
& <\frac{2^{4 n+2}+3\left(n^{2}-2 n-2\right) 2^{2 n}+2}{(n-2)\left(2^{2 n+1}-1\right)} \frac{2^{2 n+2}}{(2 n) ! \pi^{2}} \frac{2(2 n) !}{(2 \pi)^{2 n}}\left(\frac{1}{1-2^{1-2 n}}\right)-\frac{2^{2 n}}{6(n-2) \pi^{2 n-2}} \\
& :=-\frac{1}{6} \frac{2^{2 n}}{\pi^{2 n+2}\left(2 \times 2^{2 n}-1\right)\left(2^{2 n}-2\right)(n-2)} s_{n}
\end{aligned}
$$

where

$$
s_{n}=\left(2 \pi^{4}-192\right) 2^{4 n}-\left(144 n^{2}-288 n+5 \pi^{4}-288\right) 2^{2 n}+2\left(\pi^{4}-48\right) .
$$

The facts that $s_{5}=2092034 \pi^{4}-203243616>0$ and

$$
s_{n+1}-16 s_{n}=12 \cdot 2^{2 n}\left(144 n^{2}-384 n+5 \pi^{4}-240\right)+7680-160 \pi^{4}>0
$$

for $n \geqslant 3$ reveal that $s_{n}>0$ for $n \geqslant 5$, which implies that $w_{n}<0$ for $n \geqslant 5$.

A simple check shows that

$$
\begin{aligned}
& w_{3}=-\frac{2}{105 \pi^{4}}\left(17 \pi^{4}-224 \pi^{2}+560\right)<0, \\
& w_{4}=-\frac{4}{2835 \pi^{6}}\left(124 \pi^{6}-1377 \pi^{4}+15120\right)<0,
\end{aligned}
$$

which proves the decreasing property of $h(x)$ on $(0, \pi / 2)$. In view of

$$
\lim _{x \rightarrow(\pi / 2)^{-}} h(x)=\frac{8}{3 \pi^{2}}\left(\pi^{4}-96\right), \lim _{x \rightarrow 0^{+}} h(x)=\frac{8}{15} \pi^{2}\left(10-\pi^{2}\right),
$$

we conclude that

$$
\frac{8}{3 \pi^{2}}\left(\pi^{4}-96\right)<\frac{\frac{2}{3} \pi^{4} x^{3}-\left(\pi^{2}-4 x^{2}\right)^{2}\left(x \sec ^{2} x-\tan x\right)}{x^{5}}<\frac{8}{15} \pi^{2}\left(10-\pi^{2}\right),
$$

which proves the desired inequalities. 


\section{The Proof of Theorem 1.6}

We first consider the function

$$
f(x)=\left(x \sec ^{2} x-\tan x\right)-\frac{\frac{160 \pi^{4}-32 \pi^{6}+15360}{15 \pi^{4}} x^{7}+\frac{2 \pi^{4}}{3} \chi^{3}+\left(\frac{8 \pi^{4}}{15}-\frac{16 \pi^{2}}{3}\right) x^{5}}{\pi^{4}\left(1-\frac{4 x^{2}}{\pi^{2}}\right)^{2}} .
$$

Expanding in power series gives

$$
\begin{aligned}
x \sec ^{2} x-\tan x=x^{3} \sum_{n=2}^{\infty} \frac{(n-1)\left(2^{2 n}-1\right) 2^{2 n+1}}{(2 n) !}\left|B_{2 n}\right| x^{2 n-4}, \\
\frac{\frac{160 \pi^{4}-32 \pi^{6}+15360}{15 \pi^{4}} x^{7}+\frac{2 \pi^{4}}{3} x^{3}+\left(\frac{8 \pi^{4}}{15}-\frac{16 \pi^{2}}{3}\right) x^{5}}{\pi^{4}\left(1-\frac{4 x^{2}}{\pi^{2}}\right)^{2}}=\frac{2}{3} x^{3}+x^{3} \sum_{n=1}^{\infty}\left(64 n-64+\frac{2}{15} \pi^{6}\right) \frac{2^{2 n}}{\pi^{2 n+4}} x^{2 n} \\
=\frac{2}{3} x^{3}+x^{3} \sum_{n=3}^{\infty}\left(64 n-128-64+\frac{2}{15} \pi^{6}\right) \frac{2^{2 n-4}}{\pi^{2 n}} x^{2 n-4},
\end{aligned}
$$

which yields

$$
f(x)=x^{3} \sum_{n=3}^{\infty} a_{n} x^{2 n-4}
$$

where

$$
a_{n}=\frac{(n-1)\left(2^{2 n}-1\right) 2^{2 n+1}}{(2 n) !}\left|B_{2 n}\right|-\left(64 n-192+\frac{2}{15} \pi^{6}\right) \frac{2^{2 n-4}}{\pi^{2 n}} .
$$

We now prove $a_{n} \leqslant 0$ for $n \geqslant 3$. A check leads to $a_{3}=0$,

$$
\begin{aligned}
& a_{4}=-\frac{2}{105 \pi^{8}}\left(112 \pi^{6}-17 \pi^{8}+53760\right)<0, \\
& a_{5}=-\frac{16}{2835 \pi^{10}}\left(1512 \pi^{6}-31 \pi^{10}+1451520\right)<0 .
\end{aligned}
$$

For $n \geqslant 6$, application of the right-hand side inequality of (2.1) gives

$$
\begin{aligned}
a_{n} & =\frac{(n-1)\left(2^{2 n}-1\right) 2^{2 n+1}}{(2 n) !}\left|B_{2 n}\right|-\left(64 n-192+\frac{2}{15} \pi^{6}\right) \frac{2^{2 n-4}}{\pi^{2 n}} \\
& <\frac{(n-1)\left(2^{2 n}-1\right) 2^{2 n+1}}{(2 n) !} \frac{2(2 n) !}{(2 \pi)^{2 n}}\left(\frac{1}{1-2^{1-2 n}}\right)-\left(64 n-192+\frac{2}{15} \pi^{6}\right) \frac{2^{2 n-4}}{\pi^{2 n}} \\
& =-\frac{1}{120} \frac{2^{2 n}}{\pi^{2 n}\left(2^{2 n}-2\right)} b_{n}
\end{aligned}
$$

where

$$
b_{n}=2^{2 n}\left(\pi^{6}-960\right)-\left(480 n-2400+2 \pi^{6}\right) .
$$

It is easy to verify that $b_{n}$ satisfies the recurrence relation

$$
b_{n+1}-4 b_{n}=1440 n+6 \pi^{6}-7680>0
$$


for $n \geqslant 6$. This together with $b_{6}=4094 \pi^{6}-3932640>0$ indicates that $b_{n}>0$ for $n \geqslant 6$. It then follows that $a_{n}<0$ for $n \geqslant 6$, which proves $f(x)<0$ for $x \in(0, \pi / 2)$. Since

$$
\lim _{x \rightarrow(\pi / 2)^{-}} \frac{\left(\pi^{2}-4 x^{2}\right)^{2}\left(x \sec ^{2} x-\tan x\right)-\left(\frac{2 \pi^{4}}{3} x^{3}+\left(\frac{8 \pi^{4}}{15}-\frac{16 \pi^{2}}{3}\right) x^{5}\right)}{\frac{160 \pi^{4}-32 \pi^{6}+15360}{15 \pi^{4}} x^{7}}=1
$$

the upper bound is sharp.

We now come to the left-hand side inequality in Theorem 1.6. Consider the function

$$
g(x)=\left(x \sec ^{2} x-\tan x\right)-\frac{\frac{34 \pi^{4}-448 \pi^{2}+1120}{105} x^{7}+\frac{2 \pi^{4}}{3} x^{3}+\left(\frac{8 \pi^{4}}{15}-\frac{16 \pi^{2}}{3}\right) x^{5}}{\pi^{4}\left(1-\frac{4 x^{2}}{\pi^{2}}\right)^{2}} .
$$

It is easy to see that

$$
\begin{aligned}
x \sec ^{2} x-\tan x & =x^{3} \sum_{n=2}^{\infty} \frac{(n-1)\left(2^{2 n}-1\right) 2^{2 n+1}}{(2 n) !}\left|B_{2 n}\right| x^{2 n-4} \\
& =\frac{2}{3} x^{3}+\sum_{n=3}^{\infty} \frac{(n-1)\left(2^{2 n}-1\right) 2^{2 n+1}}{(2 n) !}\left|B_{2 n}\right| x^{2 n-1}, \\
& \frac{34 \pi^{4}-448 \pi^{2}+1120}{105} x^{7}+\frac{2 \pi^{4}}{3} x^{3}+\left(\frac{8 \pi^{4}}{15}-\frac{16 \pi^{2}}{3}\right) x^{5} \\
=\frac{2}{3} x^{3}+\sum_{n=3}^{\infty} 2^{2 n-7} \frac{\left(1-\frac{4 x^{2}}{\pi^{2}}\right)^{2}}{\left.105 \cdot \pi^{2}-112\right) n-51 \pi^{2}+448} & \pi^{2 n-1} .
\end{aligned}
$$

Thus

$$
\begin{aligned}
g(x) & =\sum_{n=3}^{\infty} 2^{2 n-7}\left(\frac{256(n-1)\left(2^{2 n}-1\right)}{(2 n) !}\left|B_{2 n}\right|-\frac{\left(17 \pi^{2}-112\right) n-51 \pi^{2}+448}{105 \cdot \pi^{2 n-6}}\right) x^{2 n-1} \\
& :=x^{3} \sum_{n=3}^{\infty} 2^{2 n-7} c_{n} x^{2 n-4}
\end{aligned}
$$

where

$$
c_{n}=\frac{256(n-1)\left(2^{2 n}-1\right)}{(2 n) !}\left|B_{2 n}\right|-\frac{\left(17 \pi^{2}-112\right) n-51 \pi^{2}+448}{105 \cdot \pi^{2 n-6}} .
$$

We now prove $c_{n} \geqslant 0$ for $n \geqslant 3$. A check leads to $c_{3}=c_{4}=0$, and

$$
c_{5}=(62 / 2835)-\left(34 \pi^{2}-112\right) /\left(105 \pi^{4}\right) \approx 1.1105 \times 10^{-5}>0 .
$$

For $n \geqslant 6$, using the left-hand side inequality of (2.1) one can obtain

$$
\begin{aligned}
c_{n} & >\frac{256(n-1)\left(2^{2 n}-1\right)}{(2 n) !} \frac{2(2 n) !}{(2 \pi)^{2 n}}-\frac{\left(17 \pi^{2}-112\right) n-51 \pi^{2}+448}{105 \cdot \pi^{2 n-6}} \\
& =\frac{1}{105(2 \pi)^{2 n}} d_{n},
\end{aligned}
$$


where

$$
d_{n}=\left(\left(53760+112 \pi^{6}-17 \pi^{8}\right) n+51 \pi^{8}-448 \pi^{6}-53760\right) 2^{2 n}-53760(n-1) .
$$

Applying the same method to $b_{n}$ we can prove that $d_{n}>0$ for $n \geqslant 6$. This leads to $g(x)>0$ for $x \in(0, \pi / 2)$. Since

$$
\lim _{x \rightarrow 0^{+}} \frac{\left(\pi^{2}-4 x^{2}\right)^{2}\left(x \sec ^{2} x-\tan x\right)-\left(\frac{2 \pi^{4}}{3} x^{3}+\left(\frac{8 \pi^{4}}{15}-\frac{16 \pi^{2}}{3}\right) x^{5}\right)}{\frac{34 \pi^{4}-448 \pi^{2}+1120}{105} x^{7}}=1,
$$

the lower bound is sharp.

The proof of Theorem 1.6 is complete.

Remark 4.1. Theorem 1.6 can be proved using the methods and algorithms proposed in [12] and [7]. Some of the open problems have been proved by these methods in [9] and [10].

\section{A Conjecture}

In the last section we pose a conjecture as follows.

Conjecture 5.1. Let $x \in(0, \pi / 2), N$ be a natural number, $B_{2 n}$ be the even-indexed Bernoulli numbers, and for $n=1,2, \cdots$ :

$$
k_{n}=\frac{2^{2 n}}{(2 n+2) !} l_{n}
$$

where

$$
\begin{aligned}
l_{n}= & \left(2^{2 n}-4\right)(2 n-4)(2 n+2)(2 n+1)(2 n)(2 n-1)\left|B_{2 n-2}\right| \\
& -8 \pi^{2}\left(2^{2 n}-1\right)(2 n-2)(2 n+2)(2 n+1)\left|B_{2 n}\right| \\
& +4 \pi^{4}\left(2^{2 n+2}-1\right)(2 n)\left|B_{2 n+2}\right| .
\end{aligned}
$$

Then the double inequality

$$
\frac{\sum_{n=1}^{N} k_{n} x^{2 n+1}+\lambda x^{2 N+3}}{\left(\pi^{2}-4 x^{2}\right)^{2}}<x \sec ^{2} x-\tan x<\frac{\sum_{n=1}^{N} k_{n} x^{2 n+1}+\mu x^{2 N+3}}{\left(\pi^{2}-4 x^{2}\right)^{2}}
$$

holds with the best possible constants

$$
\lambda=k_{N+1}, \mu=\left(\frac{2}{\pi}\right)^{2 N+3}\left(8 \pi^{3}-\sum_{n=1}^{N} k_{n}\left(\frac{\pi}{2}\right)^{2 n+1}\right) .
$$

Obviously, the cases $N=1$ and 2 in Conjecture 5.1 give Problem 1.5 by Sun and Zhu [17] and Theorem 1.6 respectively.

Remark 5.2. We put forward a preliminary idea that one can try to use the Key theorem in Wu and Debnath [19] to complete the proof of the conjecture because we find that recently using this Theorem Wu-Debnath an improvement in the number of inequalities was obtained [8, 11, 13-15].

\section{Acknowledgment}

This paper is supported by the Natural Science Foundation of China grants No. 11471285 and the Natural Science Foundation of China grants No. 61772025. 


\section{References}

[1] M. Abramowitz, I. A. Stegun, Handbook of Mathematical Functions: with Formulas, Graphs, and Mathematical Tables, Dover publications, New York, (1972). 2.2

[2] G. Bach, Trigonometric inequality, Amer. Math. Mon., 87 (1980), 62-62. 1

[3] M. Biernacki, J. Krzyz, On the monotonicity of certain functionals in the theory of analytic functions, Ann. Univ. M. Curie-Sklodowska, 9 (1955), 135-147. 2.1

[4] C.-P. Chen, R. B. Paris, Series representations of the remainders in the expansions for certain trigonometric and hyperbolic functions with applications, arXiv, 2016 (2016), 28 pages. 1

[5] H.-F. Ge, New Sharp Bounds for the Bernoulli numbers and refinement of Becker-Stark inequalities, J. Appl. Math., 2012 (2012), 7 pages. 1

[6] A. Jeffrey, Handbook of Mathematical Formulas and Integrals, Third edition, Elsevier Academic Press, San Diego, CA, (2004). 2

[7] T. Lutovac, B. Maleševic, C. Mortici, The natural algorithmic approach of mixed trigonometric-polynomial problems, J. Inequal. Appl., 2017 (2017), 16 pages. 4.1

[8] T. Lutovac, B. Malešević, M. Rašajski, A new method for proving some inequalities related to several special functions, arXiv, 2018 (2018), 15 pages. 5.2

[9] B. Malešević, B. Banjac, I. Jovović, A proof of two conjectures of Chao-Ping Chen for inverse trigonometric functions, J. Math. Inequal., 11 (2017), 151-162. 4.1

[10] B. Malešević, T. Lutovac, B. Banjac, A proof of an open problem of Yusuke Nishizawa for a power-exponential function, arXiv, 2016 (2016), 11 pages. 4.1

[11] B. Malešević, T. Lutovac, M. Rašajski, C. Mortici, Extensions of the natural approach to refinements, and generalizations of some trigonometric inequalities, Adv. Difference Equ., 2018 (2018), 15 pages. 5.2

[12] B. Malešević, M. Makragić, A method for proving some inequalities on mixed trigonometric polynomial functions, J. Math. Inequal., 10 (2016), 849-876. 1, 4.1

[13] B. Malešević, M. Rašajski, T. Lutovac, A new approach to the sharpening and generalizations of Shafer-Fink and Wilker type inequalities, arXiv, 2017 (2017), 11 pages. 5.2

[14] B. Malešević, M. Rašajski, T. Lutovac, Refinements and generalizations of some inequalities of Shafer-Fink's type for the inverse sine function, J. Inequal. Appl., 2017 (2017), 9 pages.

[15] M. Nenezic, L. Zhu, Some improvements of Jordan-Steckin and Becker-Stark inequalities, arXiv, 2018 (2018), 12 pages. 5.2

[16] F. Qi, A double inequality for ratios of the Bernoulli numbers, ResearchGate Dataset, 2015 (2015), 6 pages. 2.3

[17] Z. Sun, L. Zhu, Some refinements of inequalities for circular functions, J. Appl. Math., 2011 (2011), 9 pages. 1, 1, 5

[18] R. R. Wilson, M. W. Ecker, M. C. Papenfuss, V. Pambuccian, H. S. Witsenhausen, P. M. Gibson, Problems and Solutions: Elementary Problems: E2737-E2742, Amer. Math. Monthly, 85 (1978), 764-765. 1

[19] S. Wu, L. Debnath, A generalization of L'Hospital-type rules for monotonicity and its application, Appl. Math. Lett., 22 (2009), 284-290. 5.2 